H. L. Segal and J. S. Watson ${ }^{4}$ by using rather cumbersome equipment succeeded in taking photographs in colour through a flexible instrument, and R. S. Nelson ${ }^{5}$ was able to improve and simplify the apparatus. A new principle in gastroscopic photography was introduced by C. Debray and P. Housset, ${ }^{6}$ who incorporated an electronic flash in the head of the instrument to produce a momentary but brilliant source of light, and by synchronizing this with the operation of a camera achieved excellent photographs.

In contrast to the photographic recording of lesions seen through a gastroscope, the idea of using "blind" gastric photography as a separate diagnostic approach was revived in Vienna in 1930,' and in the past ten years there have been several reports from Japan. ${ }^{8-10}$ The miniature Japanese gastrocamera is easily introduced and is capable of producing clear images in colour over a wide area within the stomach, only the fundus remaining inaccessible. In a study of the use of the gastrocamera in 1,100 cases Y. Hara and his colleagues $^{11}$ were able to view some small and superficial lesions not detected by $x$-ray examination. In addition the ease with which an examination could be repeated meant that it was possible to follow the course of lesions in the stomach. The gastrocamera is unlikely to provide a substitute for methods of gastric investigation in common use, but in certain cases it may well offer a valuable complementary approach.

\section{Fibrosing Alveolitis}

In a series of papers L. Hamman and A. R. Rich ${ }^{1-3}$ described four patients who rapidly became ill with increasing dyspnoea and died within six months of the onset of symptoms. Histological examination of the lungs showed widespread fibrosis of the walls of the alveoli. The condition soon became known as the Hamman-Rich syndrome, but, as so often happens when proper names are given to diseases, subsequent publications included variants which were not mentioned in the original descriptions. Thus most reported cases have had a clinical course of years rather than months, as might have been expected from the apparent chronicity of the histological features. J. G. Scadding has suggested that the acuteness of the disease and the rate at which it progresses are inversely proportional to the age at onset.4

Men at or past middle age are most often affected. Other features which have been noted include clubbing of the fingers, fever, an associated cancer of the lung, spontaneous pneumothorax, rheumatoid arthritis, hepato-splenomegaly, hypergammaglobulinaemia, hypertrophic pulmonary osteoarthropathy, and polycythaemia. Death usually occurs within two to six years from cardiac or respiratory failure. We are thus far removed from a simple syndrome. Nor can we be certain that it has a single cause. The only feature that the cases have in common is a partly inflammatory but predominantly fibrotic thickening of the walls of the aveoli of the lungs. Many attempts have been made to devise a suitable descriptive term. Diffuse idiopathic interstitial

\footnotetext{
1 Hamman, L., and Rich, A. R., Int. Clin., 1933, 1, 197.

— Trans. Amer. clin. climat. Ass., 1935, 51, 154.

3 - Bull. Johns Hopk. Hosp., 1944, 74, 177.

- Scadding, J. G., Brit. med. F., 1960, 1, 443.

5 Livingstone, J. L., Lewis, J. G., Reid, L., and Jefferson, K. E., Quart. 7 . Med., 1964, 33, 71 .
}

pulmonary fibrosis refers only to the end result, and so Scadding has suggested the name fibrosing alveolitis to indicate a disease primarily of the alveolar walls, as opposed to an organizing pneumonia in which an exudate within the alveoli becomes converted into fibrous tissue. Nevertheless, the term Hamman-Rich syndrome is still appropriate for patients who have a rapidly fatal illness.

Fibrosing alveolitis has now been reported from all over the world, with over 100 histologically proved examples since 1950 . J. L. Livingstone and his colleagues $^{5}$ have recently contributed an exhaustive review based on a clinical, radiological, and pathological study of 45 patients. They confirm that the outstanding clinical features are progressive dyspnoea, a dry cough, and persistent rales at the bases of the lungs, usually with loss of weight and clubbing of the fingers sooner or later. Perhaps the most valuable part of the review is the correlation between the radiological and pathological findings, since with serial radiographs over months or years in many of the patients the evolution of the disease could be clearly seen. The commonest and earliest radiological abnormality is a mottling, with discrete shadows up to $2 \mathrm{~mm}$. starting in the lower zones and spreading upwards, often becoming densest in the periphery of the middle and upper zones. Sometimes the mottling is fine and confluent and resembles ground glass. As the disease progresses the mottling becomes coarser, and translucencies, up to $5 \mathrm{~mm}$. but mainly less than $2 \mathrm{~mm}$. in diameter, appear within the shadows. These changes correspond with the histological stages. At first the alveolar walls are thickened by oedema, chronic inflammatory cells, and dilatation of the capillaries. Later the alveolar spaces usually contain a cellular exudate which may absorb or cause fibrosis in the spaces, or may retract and thereby contribute to the fibrosis in the walls. ${ }^{4}$ The foci are irregularly distributed through the lungs, generally being most advanced towards the bases. Sometimes a large volume of lung (such as a secondary lobule or more) is involved in the fibrosis and contains dilated cystic spaces.

The clinical and radiological diagnosis of fibrosing alveolitis is often sufficiently obvious for biopsy of the lung to be unnecessary. The possibility of effective treatment then arises. Some patients, perhaps found on routine chest radiography, have no symptoms. Others have symptoms which are not getting worse or which are progressing only very slowly. For both these groups no specific therapy is recommended. Occasionally corticosteroid drugs have been beneficial, presumably by assisting in the dispersal of exudative and inflammatory foci. The latter may be suspected when the radiographs suggest that the stage of fine mottling predominates ; mottling sometimes resolves with treatment, at least for as long as the drugs are continued. In practice steroid drugs are usually reserved for patients with rapidly progressive symptoms in the hope that the process will be halted. Simple bronchodilator drugs may reduce breathlessness when reversible obstruction of the airways is also present, while the appropriate antibiotics are indicated for purulent bronchitis, which is sometimes a feature of the later stages.

This week we print the first of a series of monthly articles on technical aspects of motoring. "Why Disk Brakes ?" is the title of the first article (see p. 517). 